\title{
Synthesis and Application of r-rhuEPO/NiO/CNTs modified Glassy Carbon Electrode as Sensor for Determination of Erythropoietin in the Blood of Athlete
}

\author{
Furong Duan \\ Department of Physical Education Teaching, Sichuan Vocational \& Technical College, Suining, \\ 629000, China \\ E-mail: duanfurong323@163.com
}

Received: 3 September 2021 / Accepted: 9 October 2021 / Published: 10 November 2021

\begin{abstract}
The goal of this research was to create an electrochemical biosensor based on reduced-rhuEPO on $\mathrm{NiO}$ nanoparticles ( $\mathrm{NiO} \mathrm{NPs}$ ) decorated carbon nanotubes (r-rhuEPO/NiO/CNTs) for easy rhuEPO determination in human blood plasma. For synthesis of electrochemical biosensor, the activated CNTs and NiO NPs were electrodeposited on GCE surface, respectively. The chronoamperometry was used for reduction rhuEPO and modified NiO/CNTs/GCE with reduced-rhuEPO (rrhuEPO/NiO/CNTs/GCE). The results of structural analyses using SEM and XRD showed that NiO NPs were heterogeneously decorated on the surface of CNTs/GCE which formed highly porous and large active surface area which enhanced the electrochemical activity of electrodes and charge transfer rate. The electrochemical studies using DPV technique showed that r-rhuEPO/NiO/CNTs/GCE was sensitive and stable rhuEPO biosensor with linear range of 100-1300 ng/l and limit of detection of $0.02 \mathrm{ng} / \mathrm{l}$. The applicability of the r-rhuEPO/NiO/CNTs/GCE as rhuEPO biosensor in athlete's blood plasma as a real sample was investigated and results indicated the obtained values for recovery $(95.66 \%$ to $99.50 \%)$ and RSD (2.12\% to $3.33 \%)$ were acceptable, and the proposed method can applied as practical rhuEPO biosensor for blood plasma samples.
\end{abstract}

Keywords: CNTs; NiO nanoparticles; Electrodeposition; Erythropoietin; Differential pulse voltammetry

\section{FULL TEXT}

(C) 2021 The Authors. Published by ESG (www.electrochemsci.org). This article is an open access article distributed under the terms and conditions of the Creative Commons Attribution license (http://creativecommons.org/licenses/by/4.0/). 